\title{
The yin and yang of idiopathic pulmonary fibrosis
}

\author{
Michael Kreuter ${ }^{1}$ and Vincent Cottin $\mathbb{1}^{2}$
}

\begin{abstract}
Affiliations: ${ }^{1}$ Center for interstitial and rare lung diseases, Pneumology and respiratory critical care medicine, Thoraxklinik, University of Heidelberg, Heidelberg, Germany and Translational Lung Research Center Heidelberg, Member of the German Center for Lung Research (DZL). ${ }^{2}$ Dept of Pulmonary Medicine and National Reference Center for Rare Pulmonary Diseases, Competence Center for Pulmonary Arterial Hypertension, Louis Pradel Hospital, Hospices Civils de Lyon, Claude Bernard Lyon 1 University, Lyon, France.

Correspondence: Michael Kreuter, Center for interstitial and rare lung diseases, Pneumology and respiratory critical care medicine, Thoraxklinik, University of Heidelberg, and Translational Lung Research Center Heidelberg, Member of the German Center for Lung Research (DZL), Röntgenstr. 1, 69126 Heidelberg, Germany. E-mail: kreuterवuni-heidelberg.de
\end{abstract}

IPF registries and clinical trial data complement each other http://ow.ly/Te9C307KonP

Cite this article as: Kreuter M, Cottin V. The yin and yang of idiopathic pulmonary fibrosis. Eur Respir J 2017; 49: 1602316 [https://doi.org/10.1183/13993003.02316-2016].

125 years ago, OsLer [1] summarised in his medical textbook for the first time the knowledge at that time on "chronic interstitial pneumonia" as pulmonary diseases with fibroid changes, easy to diagnose but difficult to classify. In his very detailed report (recommended reading for everyone interested in interstitial lung diseases (ILDs), although it probably did not correspond to idiopathic pulmonary fibrosis (IPF), as discussed elsewhere [2]), he mentioned aetiology, histopathology and clinical characteristics, and finally about treatment stated that "nothing can be done for the condition itself". Typical for this time, information for this interesting chapter was gathered mainly from reports by pathologists and from clinical observations by Osler himself and his colleagues. It took decades until further descriptions on these diseases became available, first in the report by HAMMAN and RicH [3] on patients with fatal outcomes of unexplained interstitial pneumonias, and then in the diverse classifications of ILDs beginning in the 1960s [4-6]. Almost every piece of information on clinical aspects of patients with ILDs (mainly with IPF) gathered until then and even until almost the end of the last century came from clinical observational reports, i.e. casuistics or case series, or from retrospective analyses [6].

With the first international consensus statement on IPF and the new era of pharmacological studies, our knowledge of the disease, its behaviour and finally of its treatment possibilities grew exponentially [4-7]. Post hoc analyses of large international trials, for example, allowed us to learn more about disease behaviour [8], effect of treatment of comorbid conditions on outcomes in IPF [9-11], and possible impact of radiological patterns on further diagnostic procedures [12] or on health-related quality of life, just to mention a few [13]. Yet, as clinical trials mainly recruit patients with fewer comorbidities or even exclude some comorbid conditions, completed trials have enrolled patients with milder impairment of pulmonary function, younger patients and those whose general condition, health-related quality of life and disease severity were often less pronounced, so they may possibly not reflect the real-life situation [14]. Therefore, the generalisability of these data to the entire population of patients with IPF is thought to be restricted.

This leaves us with several open questions. How can we address the patients' demands for all-encompassing information on their disease, as summarised in a recent European IPF Patient Charter [15]? How can we gain new insights into the burden of this devastating disease, including new patient-related outcomes [16],

Received: Nov 242016 | Accepted: Nov 252016

Conflict of interest: Disclosures can be found alongside this article at erj.ersjournals.com

Copyright @ERS 2017 
healthcare-associated costs, quality of life and others? Which factors may predict disease behaviour in the real world setting outside the "artificial" world of clinical trials? How can we recognise gaps in adherence to clinical guidelines and how are patients treated under real-life conditions? And finally, how can we identify open questions in treatment issues?

In this context, disease registries are increasingly recognised as important sources for collecting "real-world" information on practical issues and on extended follow-up for longer-term data collection [17, 18]. To take only one example, registries often better reflect the diagnostic process as conducted in the community than clinical trials do. Already almost 10 years ago, well-known experts in the field advocated for the implementation of an international registry for IPF [19], which was renewed recently by other experts [20], in order to gain further knowledge of the disease and of differences in diagnostic and management issues throughout the world. However, several problems have prevented the installation of an international registry and information from registries has hitherto mainly been available only from Europe and North America [14].

In this issue of the European Respiratory Journal, the report by Jo et al. [21] on the baseline characteristics of patients with IPF from the Australian IPF Registry (AIPFR) is timely. By constant efforts, our colleagues recruited as many as 647 patients with IPF. Here, they report on baseline characteristics, including comorbidities, diagnostic procedures and quality of life. Furthermore, they provide data on mortality outcomes, with a 4 -year mortality rate of $44 \%$, associations of baseline pulmonary function data to mortality and the significant, positive impact of anti-fibrotic therapy on survival.

This registry interestingly encompasses a very original process of diagnostic validation. Due to distances in Australia, a multidisciplinary discussion [22] with the physical presence of every doctor involved was not practically feasible; however, the investigators still wanted the diagnosis to undergo some central validation process. Therefore, they organised a virtual multidisciplinary discussion process, in which radiologists (in two radiology panels, with three members each) had to obtain consensus on images provided on an electronic platform, while a panel of three pathologists went through a similar process. All data were then reviewed by the single clinical panel of three IPF-expert pulmonologists who assigned an IPF Registry multidisciplinary discussion diagnosis. Whether this original process reaches a high level of consensus and reproducibility, and can be useful in other places in the world, remains to be determined. Recently, several groups have produced data about the multidisciplinary team meeting process [23], including some from the Australian registry [24], with the potential to help our practice to evolve.

The report by Jo et al. [21] has several strengths. In a country with diverse ancestries, different sources of exposures than in other regions of the world, limited access to anti-fibrotic therapies and other differences, it adds interesting information to address the need to understand IPF better throughout the world. It should also be acknowledged that these authors provided important information on patient-related outcomes, such as the quality of life and the burden of symptoms as well as (disease-associated) depression. Furthermore, it is interesting to learn that comorbidities are frequent but do not affect outcome (in contradiction to recent reports [25]), including reflux, which was, however, more common than in other registries [26].

In this context, the authors' commitment to compare their data with other recent registries and trials is very valuable. In comparison to the German INSIGHTS-IPF (Investigating Significant Health Trends in Idiopathic Pulmonary Fibrosis) registry [26], for example, AIPFR patients had less severe impairment of quality of life and better functional data. Whether this truly reflects differences between countries or continents has yet to be analysed critically but opens the book for future tasks in registries throughout the world. Only by this method will we be able to understand the ILD entity-related intercultural differences, e.g. why in India the most commonly diagnosed ILD is thought to be hypersensitivity pneumonitis $(\sim 47 \%)$, while IPF may only occur in $\sim 14 \%$ [27].

This AIPFR report also reflects the current situation in Australia on the diagnosis of IPF/ILD, as all patients registered were considered to have IPF in the analyses as diagnosed by their treating pulmonologists. Later on, diagnosis was re-evaluated by a central multidisciplinary team and patients were categorised as "definite", "probable", "possible" or "inconsistent" with IPF according to the current guidelines from the American Thoracic Society, European Respiratory Society, Japanese Respiratory Society and Latin American Thoracic Association [4]. Much to our regret, we have to wait for these insightful data as they will be part of further analyses.

Finally and most interesting are the mortality differences in patients being treated with anti-fibrotic drugs compared with those without access to these drugs, significantly favouring treatment with anti-fibrotics. However, this finding brings us to the limitations of this report. Both the anti-fibrotic drugs were available only during clinical trials, patient access programmes or private purchase. This is one example of why it is important to read registry analyses critically, as recruitment is often prone to selection bias, at least in part. 
Additionally, both prevalent and incident cases were recruited simultaneously, without differentiating clinical characteristics between these two patient subsets, which may affect survival results. Also, interested readers need to acknowledge how information was gathered, e.g. here comorbidities were self-reported as part of a structured questionnaire and therefore accuracy may be limited, which may explain differences from other reports. Finally, one of the major limitations (and an important challenge) of this and any registry was represented by missing data.

While these authors have to be commended for their tremendous contribution to the field, there are still many lessons to be learned. We would like to request all current registries to keep helping us to understand this disease better, by informing us about new developments in diagnosing IPF and analysing acute exacerbations, and providing us with real-world data on new developments in treatments, including non-pharmacological strategies. In pulmonary arterial hypertension, there are now at least half a dozen national or multinational registries, which have provided a huge amount of unexpected observations that would not have been made possible only by trials. There is reasonable hope that IPF registries will continue to inform us tremendously in the years to come.

In conclusion, as in the Chinese philosophy, we should approach IPF with seemingly opposite energies: the "yin", the more passive way to collect information on the disease through registries; and the "yang", the more focused and active part in approaching the disease by clinical trials. Learning from the real world will help transfer new ideas into clinical trials, and clinical trials will help us to answer questions that can only be addressed in a standardised, randomised fashion [28]. Only by understanding that we need both sources of information will we gain balanced information and learn how to address this disease in future. A still unsolved task is the implementation of an international registry or at least the transformation of regional registries into part of a global network. Several initiatives, sponsored by scientific societies, patient support groups and the pharmaceutical industry, have recently undertaken the task of developing meta-registries. In this way, we may soon be able to understand the disease and its diversity better, on a worldwide level.

\section{References}

$1 \quad$ Osler W. The Principles and Practice of Medicine. New York, D. Appleton and Co., 1892.

2 Cordier JF, Cottin V. Neglected evidence in idiopathic pulmonary fibrosis: from history to earlier diagnosis. Eur Respir I 2013; 42: 916-923.

3 Hamman L, Rich AR. Acute diffuse interstitial fibrosis of the lung. Bull Johns Hopkins Hosp 1944; 74: 177-212.

4 Raghu G, Collard HR, Egan JJ, et al. An official ATS/ERS/JRS/ALAT statement: idiopathic pulmonary fibrosis: evidence-based guidelines for diagnosis and management. Am J Respir Crit Care Med 2011; 183: 788-824.

5 Raghu G, Rochwerg B, Zhang Y, et al. An official ATS/ERS/JRS/ALAT clinical practice guideline: treatment of idiopathic pulmonary fibrosis. An update of the 2011 clinical practice guideline. Am J Respir Crit Care Med 2015; 192: e3-e19.

6 American Thoracic Society. Idiopathic pulmonary fibrosis: diagnosis and treatment. International consensus statement. American Thoracic Society (ATS), and the European Respiratory Society (ERS). Am J Respir Crit Care Med 2000; 161: 646-664.

7 Kreuter M, Bonella F, Wijsenbeek M, et al. Pharmacological treatment of idiopathic pulmonary fibrosis: current approaches, unsolved issues, and future perspectives. Biomed Res Int 2015; 2015: 329481.

8 Ley B, Bradford WZ, Vittinghoff E, et al. Predictors of mortality poorly predict common measures of disease progression in idiopathic pulmonary fibrosis. Am J Respir Crit Care Med 2016; 194: 711-718.

9 Kreuter M, Wijsenbeek MS, Vasakova M, et al. Unfavourable effects of medically indicated oral anticoagulants on survival in idiopathic pulmonary fibrosis. Eur Respir J 2016; 47: 1776-1784.

10 Kreuter M, Wuyts W, Renzoni E, et al. Antacid therapy and disease outcomes in idiopathic pulmonary fibrosis: a pooled analysis. Lancet Respir Med 2016; 4: 381-389.

11 Kreuter M, Bonella F, Maher TM, et al. Effect of statins on disease-related outcomes in patients with idiopathic pulmonary fibrosis. Thorax 2016; in press [https://doi.org/10.1136/thoraxjnl-2016-208819].

12 Raghu G, Lynch D, Godwin JD, et al. Diagnosis of idiopathic pulmonary fibrosis with high-resolution CT in patients with little or no radiological evidence of honeycombing: secondary analysis of a randomised, controlled trial. Lancet Respir Med 2014; 2: 277-284.

13 Swigris JJ, Brown KK, Behr J, et al. The SF-36 and SGRQ: validity and first look at minimum important differences in IPF. Respir Med 2010; 104: 296-304.

14 Harari S, Caminati A. Idiopathic pulmonary fibrosis: from clinical trials to real-life experiences. Eur Respir Rev 2015; 24: 420-427.

15 Bonella F, Wijsenbeek M, Molina-Molina M, et al. European IPF Patient Charter: unmet needs and a call to action for healthcare policymakers. Eur Respir J 2016; 47: 597-606.

16 Wijsenbeek M, van Manen M, Bonella F. New insights on patient-reported outcome measures in idiopathic pulmonary fibrosis: only PROMises? Curr Opin Pulm Med 2016; 22: 434-441.

17 Cottin V, Wuyts W. Insights into idiopathic pulmonary fibrosis in the real world. Eur Respir J 2015; 46: 16-18.

18 Kreuter M, Herth FJ, Wacker M, et al. Exploring clinical and epidemiological characteristics of interstitial lung diseases: rationale, aims, and design of a nationwide prospective registry - the EXCITING-ILD Registry. Biomed Res Int 2015; 2015: 123876.

19 Wilson JW, du Bois RM, King TE Jr. Challenges in pulmonary fibrosis: 8 . The need for an international registry for idiopathic pulmonary fibrosis. Thorax 2008; 63: 285-287.

20 Ryerson CJ, Corte TJ, Collard HR, et al. A global registry for idiopathic pulmonary fibrosis: the time is now. Eur Respir J 2014; 44: 273-276. 
21 Jo HE, Glaspole I, Grainge C, et al. Baseline characteristics of idiopathic pulmonary fibrosis: analysis from the Australian Idiopathic Pulmonary Fibrosis Registry. Eur Respir J 2017; 49: 1601592.

22 Flaherty KR, King TE Jr, Raghu G, et al. Idiopathic interstitial pneumonia: what is the effect of a multidisciplinary approach to diagnosis? Am J Respir Crit Care Med 2004; 170: 904-910.

23 Walsh SL, Wells AU, Desai SR, et al. Multicentre evaluation of multidisciplinary team meeting agreement on diagnosis in diffuse parenchymal lung disease: a case-cohort study. Lancet Respir Med 2016; 4: 557-565.

24 Jo HE, Glaspole IN, Levin KC, et al. Clinical impact of the interstitial lung disease multidisciplinary service Respirology 2016; 21: 1438-1444.

25 Kreuter M, Ehlers-Tenenbaum S, Palmowski K, et al. Impact of comorbidities on mortality in patients with idiopathic pulmonary fibrosis. PLoS One 2016; 11: e0151425.

26 Behr J, Kreuter M, Hoeper MM, et al. Management of patients with idiopathic pulmonary fibrosis in clinical practice: the INSIGHTS-IPF registry. Eur Respir J 2015; 46: 186-196.

27 Singh S, Collins BF, Sharma BB, et al. Interstitial lung disease (ILD) in India: results of a prospective registry. Am J Respir Crit Care Med 2016; in press [https://doi.org/10.1164/rccm.201607-1484OC].

28 King SB 3rd. "Real-world evidence?”: get real! JACC Cardiovasc Interv 2016; 9: 204-205. 